\title{
Effect of melatonin on the peripheral Tymphocyte cell cycle and levels of reactive oxygen species in patients with premature ovarian failure
}

\author{
YANMIN LI ${ }^{1,2}$, HONGLI LIU ${ }^{3}$, JING SUN ${ }^{4}$, YIPENG TIAN ${ }^{5}$ and CHANGZHONG LI ${ }^{1}$ \\ ${ }^{1}$ Department of Gynaecology, Shandong Provincial Hospital Affiliated to Shandong University, Jinan, Shandong 250021; \\ ${ }^{2}$ Department of Reproductive Medicine, Weifang People's Hospital; ${ }^{3}$ Department of Obstetrics, Weifang Traditional \\ Chinese Medicine Hospital; ${ }^{4}$ Department of Obstetrics, Weifang People's Hospital; ${ }^{5}$ Weifang People's Hospital, \\ Weifang, Shandong 261041, P.R. China
}

Received July 15, 2016; Accepted October 3, 2016

DOI: $10.3892 /$ etm.2016.3833

\begin{abstract}
The objective of the present study was to observe the curative effect and mechanism of melatonin for suppression of premature ovarian failure (POF). From December 2014 to June 2015, 128 patients were consecutively diagnosed with POF in the Department of Gynaecology and Obstetrics. The patients were randomly divided into the experimental and control groups. The experimental group received melatonin tablets (1-3 mg/day), while the control group received placebo tablets. The levels of six sex hormones, percentage of $\mathrm{T}$ lymphocytes in the $\mathrm{G}_{1} / \mathrm{M}$ phase, and levels of reactive oxygen species (ROS) were determined at four different time-points ( 1 day before treatment, and at 1, 3 and 6 months after treatment) in both groups. After 6 months of treatment, the levels of luteinizing hormone and follicle-stimulating hormone were significantly decreased in the experimental group compared with the control group $(\mathrm{P}<0.05)$. Compared with the control group, the levels of ROS in plasma were significantly decreased in the experimental group $(\mathrm{P}<0.05)$. Correlation analysis showed that the levels of melatonin in peripheral blood were negatively related with the levels of ROS ( $\mathrm{rs}=-0.481, \mathrm{P}<0.05)$. One-year follow-up study showed that the normal excretion of ovarian hormones in the experimental group was significantly higher than that of the control group $(\mathrm{P}<0.05)$. In conclusion, treatment with melatonin is an effective approach to suppress POF. The potential mechanism of melatonin is inhibition of ROS production and protection of the process of normal follicle development.
\end{abstract}

Correspondence to: Dr Changzhong Li, Department of Gynaecology, Shandong Provincial Hospital Affiliated to Shandong University, 324 Jingwu Road, Jinan, Shandong 250021, P.R. China E-mail: liyanmin160701@163.com

Key words: melatonin, premature ovarian failure, oxygen free radicals

\section{Introduction}

Premature ovarian failure (POF) is defined as ovarian failure which leads to amenorrhea before the age of 40 years. It is characterized by primary or secondary amenorrhea accompanied by increased blood gonadotropin levels and reduced levels of estrogen. The clinical features primarily include symptoms related to low estrogen such as depression, facial flushing, and low libido $(1,2)$. Roussev et al examined 1,858 women with natural amenorrhea and demonstrated that the incidence of POF was $<1 \%$ before the age of 40 years, and $<1 / 1,000$ in women aged under 30 years (3). In Beijing, the incidence rate of POF is $1.8 \%$ (4).

Melatonin is an amine hormone produced primarily by the pineal gland in mammals. It can regulate the reproductive activity and the photoperiod determines its biosynthesis. Previous findings showed there are many different melatonin receptors in the ovary, which suggests that melatonin has a significant role in the reproductive system (5). However, the role of melatonin in POF is not clear.

By measuring the cell cycle of $T$ lymphocytes and the levels of reactive oxygen species (ROS) in the plasma of patients with POF before and after treatment with melatonin, the aim of the present study was to determine the mechanism of melatonin for treating POF, and to provide a theoretical basis for the clinical treatment of POF.

\section{Patients and methods}

Patients. From December 2014 to June 2015, 128 patients who were diagnosed with POF in the Department of Gynaecology and Obstetrics of Shandong Provincial Hospital were randomly divided into the experimental and control groups. Patients in the experimental group received melatonin tablets (1-3 mg/day), and patients in the control group took the corresponding placebo (similar in appearance to melatonin tablets). We measured the levels of six sex hormones, cell cycle of $\mathrm{T}$ lymphocytes, and the levels of ROS in plasma of patients 1 day before treatment, and at 1, 3 and 6 months after treatment. Data were collected and analyzed. The inclusion criteria 
Table I. Comparison of baseline clinical characteristics.

\begin{tabular}{lcccc}
\hline Groups & Patients $(\mathrm{n})$ & Age $($ years $)$ & BMI $\left(\mathrm{kg} / \mathrm{m}^{2}\right)$ & Menstrual cycle (days) \\
\hline Experimental & 64 & $29.4 \pm 9.8$ & $21.5 \pm 2.4$ & $56.4 \pm 2.7$ \\
Control & 64 & $31.2 \pm 8.3$ & $20.3 \pm 1.7$ & $61.5 \pm 1.2$ \\
T-value & - & 0.22 & 0.27 & 0.98 \\
P-value & - & 0.38 & 0.71 & 0.11 \\
\hline
\end{tabular}

BMI, body mass index.

were: i) Patients were aged $>18$ years; and ii) diagnosis of POF by laboratory examination. The exclusion criteria included: i) Ovarian tumors; ii) malignant tumors of other tissues/organs; iii) diagnosis could not be made; iv) cognitive impairment or suffering from mental illness; v) blood samples could not be obtained from patients; vi) patients and their families did not match the information they previously provided; vii) patient quit the study; and viii) patients with poor general condition, or unsuitable for diagnosis and treatment.

Blood collection. Patients fasted for $8 \mathrm{~h}$ and $3 \mathrm{ml}$ blood from the elbow vein was collected in $1.8 \mathrm{ml}$ vacuum tubes containing $0.2 \mathrm{ml} \mathrm{3.8 \%} \mathrm{sodium} \mathrm{citrate.} \mathrm{Specimens} \mathrm{were} \mathrm{collected} \mathrm{within}$ $1 \mathrm{~h}$ after centrifugation $(2,500 \mathrm{x} \mathrm{g}, 10 \mathrm{~min})$. Serum or plasma was stored in $0.5 \mathrm{ml}$ Eppendorf tubes and preserved at $-30^{\circ} \mathrm{C}$. Samples collected were used within 1 month.

Reagents. DCFH-DA powder (Sigma-Aldrich, St. Louis, MO, USA), sterile double-distilled water and phospho-TGF- $\beta 1$ (p-TGF- $\beta 1)$ antibody (1:1,000; Cell Signaling Technology, Inc., Danvers, MA, USA), $\beta$-actin antibody $(1: 5,000$; Invitrogen, Carlsbad, CA, USA), 0.9\% sterile saline (Otsuka Pharmaceutical Co., Ltd., Tokyo, Japan), TRIzol (Invitrogen) and enzyme-linked immunosorbent assay (ELISA) kit (Cayman Chemical Co., Ann Arbor, MI, USA) were used.

Experimental instruments. Centrifuge and micropipettes (both from Eppendorf AG, Hamburg, Germany), Haier ice machine, western blot electrophoresis apparatus (Bio-Rad, Berkeley, CA, USA), $-80^{\circ} \mathrm{C}$ refrigerator (Thermo Fisher Scientific, Waltham, MA, USA); $10 \mathrm{ml}$ syringe, $5 \mathrm{ml}$ syringe (HA well, Tianjin), experimental animal special surgical instruments (Beijing Medical Instrument Factory, Beijing, China), NanoDrop 2000 spectrophotometer (Thermo Fisher Scientific, Wilmington, DE, USA), Eppendorf tubes (Eppendorf AG), water bath (Beijing Medical Instrument Factory), pathological tissue processor (Leica, Mannheim, Germany), and flow cytometer (CyFlow; Partec, Münster, Germany) were used in the study.

Detection of ROS and VEGF levels. To determine ROS and vascular endothelial growth factor (VEGF) levels, blood samples were centrifuged and the supernatant was used for measurements. The human serum ROS and VEGF kits were used according to the manufacturer's instructions.

Tlymphocyte extraction. i) A proper amount of lymphocyte separation liquid was added to appropriate tubes. ii) Heparin anticoagulated venous blood and an equal volume of Hank's solution or RPMI-1640 were uniformly mixed, and close attention was paid to maintain a clear interface. Samples were centrifuged in a horizontal centrifuge (1,600 x g, $20 \mathrm{~min})$. iii) Samples in the tubes were resolved into three layers; the upper layer was plasma and Hank's solution, and the lower layer was primarily red blood cells and granular cells. The middle layer was the lymphocyte separation solution. The interface of the upper and the middle layers was the Buffy coat. This layer also contained platelets. iv) The capillary was inserted into the Buffy coat to aspirate cells. Cells were placed in another short tube, and Hank's solution or RPMI-1640 was added. The samples were then centrifuged $(1,200 \mathrm{x} \mathrm{g}, 10 \mathrm{~min})$ and cells were washed twice. v) After the final centrifugation, RPMI1640 with $10 \%$ fetal bovine serum was added to the supernatant. A drop of cell suspension and a drop of $0.2 \%$ trypan blue dye were mixed. The cells were counted with a hemocytometer.

Melatonin treatment. The patients received $1 \mathrm{mg} / \mathrm{night}$ before bed service. For patients aged 41-60 years, $1-3 \mathrm{mg}$ were given every night.

Urinary oxidative stress test. After 6 weeks of melatonin treatment, urine was collected and immediately stored at $-80^{\circ} \mathrm{C}$. The levels of urinary $8-\mathrm{OHdG}$ and 8 -isoPGF2 $\alpha$ were determined by the ELISA kit. Determination of NT levels in urine were by chemiluminescence kit (Millipore Corp., Bedford, MA, USA).

Statistical analysis. SPSS 13.0 statistical software (Chicago, IL, USA) was used for data analysis. Comparisons of mean values were conducted using the t-test, and comparisons of rate were made using the $\chi^{2}$ test. The correlation between the levels of melatonin and ROS was determined by the correlation analysis and multivariate linear regression analysis. $\mathrm{P}<0.05$ was considered to indicate a statistically significant difference.

\section{Results}

Comparison of baseline clinical characteristics. The baseline clinical characteristics of the two groups including age, body mass index (BMI), and menstrual cycle were similar. There were no statistically significant differences between the two groups $(\mathrm{P}>0.05)$ (Table I).

Comparison of sex hormone levels. We treated the patients in the experimental group with melatonin for 6 months. The 
Table II. Comparison of sex hormones (pmol/l).

\begin{tabular}{|c|c|c|c|c|c|c|c|c|}
\hline Test project & Groups & Patients (n) & Before & 1 month & 3 months & 6 months & T-value & P-value \\
\hline \multirow[t]{4}{*}{ FSH } & Experimental & 64 & $45.3 \pm 3.6$ & $33.3 \pm 1.6$ & $20.4 \pm 1.6$ & $15.49 \pm 2.3$ & 24.37 & 0.004 \\
\hline & Control & 64 & $46.5 \pm 4.4$ & $47.5 \pm 2.4$ & $48.6 \pm 1.8$ & $47.49 \pm 2.3$ & 0.98 & 0.11 \\
\hline & T-value & - & 0.21 & 28.21 & 22.67 & 25.48 & - & - \\
\hline & P-value & - & 0.33 & 0.004 & 0.001 & 0.005 & - & - \\
\hline \multirow[t]{4}{*}{ LH } & Experimental & 64 & $28.4 \pm 9.1$ & $18.4 \pm 2.3$ & $15.3 \pm 3.4$ & $10.4 \pm 2.1$ & 22.43 & 0.001 \\
\hline & Control & 64 & $28.7 \pm 6.4$ & $27.8 \pm 2.1$ & $28.2 \pm 3.3$ & $27.9 \pm 0.42$ & 0.58 & 0.41 \\
\hline & T-value & - & 0.87 & 10.22 & 39.38 & 42.48 & - & - \\
\hline & P-value & - & 0.24 & 0.012 & 0.004 & 0.013 & - & - \\
\hline \multirow[t]{4}{*}{$\mathrm{E} 2$} & Experimental & 64 & $62.7 \pm 33.4$ & $89.3 \pm 12.5$ & $112.4 \pm 23.1$ & $142.7 \pm 33.4$ & 42.5 & 0.001 \\
\hline & Control & 64 & $72.4 \pm 15.8$ & $72.4 \pm 12.8$ & $79.4 \pm 35.8$ & $76.4 \pm 21.8$ & 0.48 & 0.25 \\
\hline & T-value & - & 0.47 & 12.9 & 24.9 & 31.3 & - & - \\
\hline & P-value & - & 0.52 & 0.04 & 0.02 & 0.006 & - & - \\
\hline
\end{tabular}

FSH, follicle-stimulating hormone; LH, luteinizing hormone; E2, estradiol.

Table III. Comparison of levels of ROS and VEGF.

\begin{tabular}{llccccccc}
\hline Test projects & \multicolumn{1}{c}{ Groups } & Patients & Before treatment & 1 month & 3 months & 6 months & T-value & P-value \\
\hline \multirow{2}{*}{ ROS (ng/ml) } & Experimental & 64 & $203.4 \pm 21.2$ & $167.4 \pm 12.1$ & $123.2 \pm 13.5$ & $58.5 \pm 22.7$ & 44.37 & 0.004 \\
& Control & 64 & $210.4 \pm 18.3$ & $209.8 \pm 15.6$ & $208.3 \pm 19.4$ & $211.2 \pm 13.3$ & 87.4 & 0.001 \\
& T-value & - & 0.21 & 20.35 & 44.72 & 50.53 & - & - \\
& P-value & - & 0.33 & 0.01 & 0.001 & 0.006 & - \\
VEGF (ng/ml) & Experimental & 64 & $48.5 \pm 12.6$ & $22.5 \pm 10.7$ & $12.3 \pm 2.6$ & $10.7 \pm 9.4$ & 24.3 & 0.002 \\
& Control & 64 & $51.3 \pm 10.9$ & $55.2 \pm 9.3$ & $58.4 \pm 11.5$ & $56.5 \pm 12.1$ & 0.67 & 0.33 \\
& T-value & - & 0.29 & 28.37 & 39.42 & 33.51 & - & - \\
& P-value & - & 0.77 & 0.009 & 0.005 & 0.006 & - \\
\hline
\end{tabular}

ROS, reactive oxygen species; VEGF, vascular endothelial growth factor.

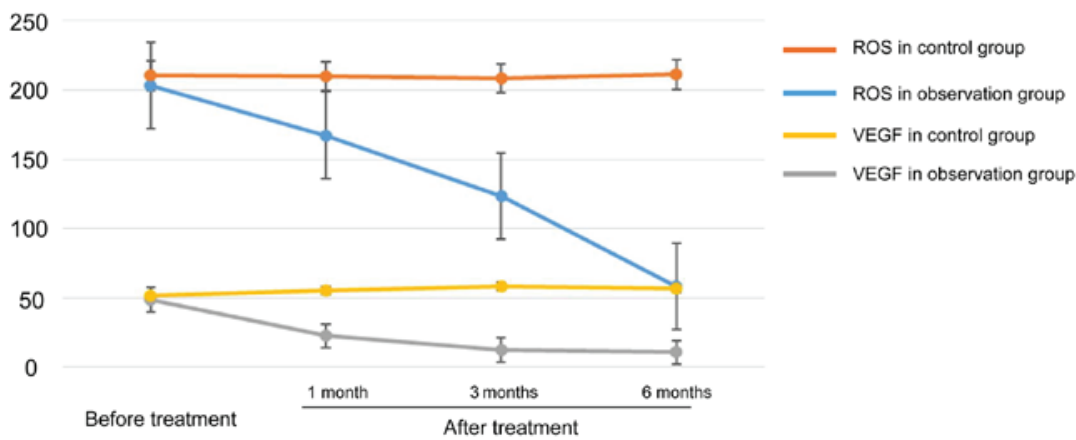

Figure 1. Changes in the levels of ROS and VEGF before and 1,3 and 6 months after treatment with melatonin in the two groups. ROS, reactive oxygen species; VEGF, vascular endothelial growth factor.

control group was treated with placebo. After 6 months of treatment, we found that the levels of follicle-stimulating hormone (FSH) and luteinizing hormone (LH) were significantly decreased in the experimental group, and the levels of estradiol (E2) were significantly increased $(\mathrm{P}<0.05)$. However, there was no significant difference in the menstrual cycle (Table II).
Comparison of plasma ROS and VEGF levels. The levels of ROS and VEGF in plasma were significantly lower in the experimental group compared with the control group $(\mathrm{P}<0.05)$ (Table III and Fig. 1).

Determination of T lymphocyte cell cycle. Peripheral blood $\mathrm{T}$ lymphocytes were extracted from the two groups of patients 
Table IV. Comparison of percentage of T lymphocytes in $\mathrm{G}_{1} / \mathrm{M}$ phase (\%).

\begin{tabular}{lccccccc}
\hline Groups & Patients & Before treatment & 1 month & 3 months & 6 months & T-value & P-value \\
\hline Experimental & 64 & $33.7 \pm 3.4$ & $22.5 \pm 1.6$ & $18.3 \pm 1.4$ & $15.5 \pm 2.5$ & 44.37 & 0.004 \\
Control & 64 & $38.4 \pm 2.7$ & $37.3 \pm 2.4$ & $38.6 \pm 2.7$ & $37.6 \pm 3.2$ & 0.44 & 0.507 \\
T-value & - & 0.21 & 22.7 & 31.5 & 34.7 & - & - \\
P-value & - & 0.33 & 0.01 & 0.008 & 0.007 & - & - \\
\hline
\end{tabular}

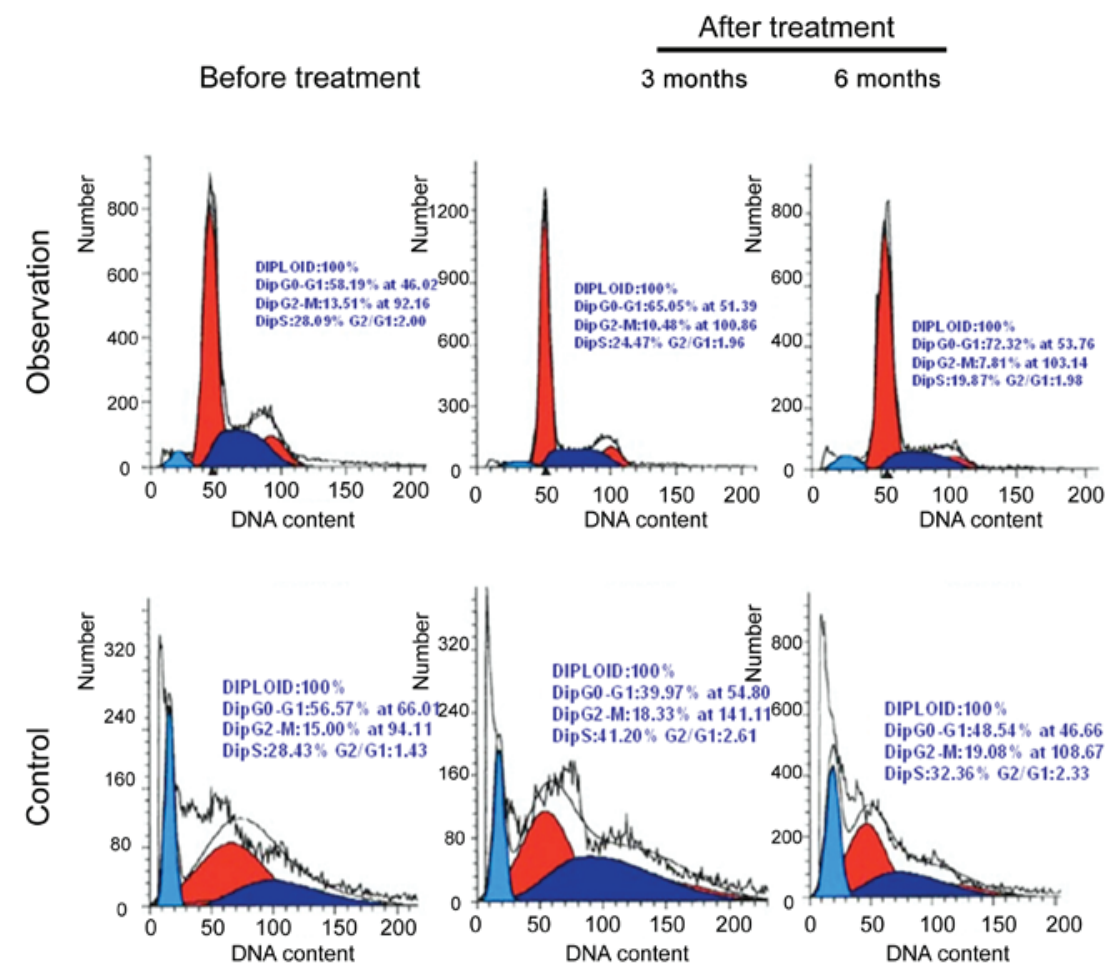

Figure 2. Detection of T lymphocyte cell cycle before and after treatment with melatonin.

Table V. Measurement of urinary oxidative stress.

\begin{tabular}{lcccccc}
\hline & & \multicolumn{2}{c}{$8-\mathrm{OHdG}$} & & \multicolumn{2}{c}{8 -isoPGF2 $\alpha$} \\
Groups & Patients (n) & Before treatment & After treatment & & Before treatment & After treatment \\
\hline Experimental & 64 & $15.7 \pm 3.4$ & $11.5 \pm 1.6^{\mathrm{a}}$ & & $14.3 \pm 1.4$ & $11.5 \pm 2.5^{\mathrm{b}}$ \\
Control & 64 & $15.4 \pm 2.7$ & $16.3 \pm 2.4$ & & $14.6 \pm 2.7$ & $15.6 \pm 3.2$ \\
T-value & - & 0.11 & 21.7 & & 36.5 & 34.2 \\
P-value & - & 0.83 & 0.01 & & 0.008 & 0.007 \\
\hline
\end{tabular}

${ }^{\mathrm{a} C}$ Compared with the results before treatment, $\mathrm{T}=12.5, \mathrm{P}<0.05 .{ }^{\mathrm{b}}$ Compared with the results before treatment, $\mathrm{T}=21.3, \mathrm{P}<0.05$.

before treatment, and after 1, 3 and 6 months of treatment. The $\mathrm{T}$ lymphocyte cell cycle was measured. The number of cells in the $\mathrm{G}_{1} / \mathrm{M}$ phase in the experimental group was less than that in the control group, and the difference was statistically significant $(\mathrm{P}<0.05)$ (Table IV and Fig. 2).

Urinary oxidative stress test. Urinary oxidative stress levels were measured before and after treatment in the two
Table VI. Comparison of ovulation.

\begin{tabular}{lccc}
\hline Groups & Patients & Positive & Negative \\
\hline Experimental & 64 & 18 & 46 \\
Control & 64 & 0 & 64 \\
$\chi^{2}$ value & - & 12.83 & \\
P-value & - & 0.011 & \\
\hline
\end{tabular}


Table VII. Correlation analysis between ROS level and clinical characteristics of patients.

\begin{tabular}{lccccccccc}
\hline Melatonin & Gender & Age (years) & FSH & LH & E2 & Periods & ROS & BMI $\left(\mathrm{kg} / \mathrm{m}^{2}\right)$ \\
\hline $\mathrm{r}$ & 0.021 & 0.351 & 0.172 & 0.151 & 0.064 & 0.382 & -0.481 & 0.141 \\
P-value & $>0.05$ & $>0.05$ & $>0.05$ & $>0.05$ & $<0.05$ & $>0.05$ & $<0.05$ & $<0.05$ \\
\hline
\end{tabular}

ROS, reactive oxygen species; FSH, follicle-stimulating hormone; LH, luteinizing hormone; E2, estradiol; BMI, body mass index.

Table VIII. Multivariate linear regression analysis.

\begin{tabular}{lccccccr}
\hline Variables & & & & & \multicolumn{2}{c}{$(95 \% \mathrm{CI})$} \\
\cline { 5 - 8 } & $\beta$ & $\mathrm{SE}$ & $\beta^{\prime}$ & $\mathrm{t}$ & P-value & Upper & lower \\
BMI & 0.531 & 0.14 & 0.764 & 0.412 & $>0.05$ & 0.26 & 0.81 \\
E2 & 0.581 & 0.10 & 0.642 & 0.652 & $>0.05$ & 0.39 & 0.78 \\
ROS & 0.768 & 0.08 & 0.871 & 0.981 & $<0.05$ & 0.61 & 0.92 \\
\hline
\end{tabular}

BMI, body mass index; E2, estradiol; ROS, reactive oxygen species; CI, confidence interval.

groups. The results showed that after treatment, the levels of urinary 8 -OHdG and 8 -isoPGF2 $\alpha$ were significantly lower in the experimental group compared with the control group $(\mathrm{P}<0.05)($ Table V).

Comparison of ovulation. We compared ovulation in the two groups of patients after treatment and found that compared with the control group, there were significantly more patients with ovulation in the experimental group after treatment $(\mathrm{P}<0.05)$ (Table VI).

Correlation analysis of serum melatonin and ROS. We performed a correlation analysis of melatonin and the levels of ROS. We found a negative correlation between serum melatonin level and the levels of ROS, and the difference was statistically significant $(\mathrm{P}<0.05)$, correlation coefficient rs $=-0.481$ (Tables VII and VIII).

\section{Discussion}

Melatonin (N-acetyl-5-methoxytryptamine, MT) is a neuroendocrine hormone secreted by the pineal gland. Recent studies have indicated that it plays a significant role in the reproductive process (6-9). Human sinus follicular fluid contains a high concentration of MT. The surface of ovarian tissue granulosa cells also express the MT receptor, which indicates that MT can directly regulate ovarian function through a variety of ways. Findings have shown that in many patients with POF, ROS participate in normal follicular development at each physiological stage including: Folliculogenesis and atresia, ovulation, oocyte maturation, and corpus luteum formation (10). MT and its metabolites are considered to be potent antioxidants and free radical scavengers which prevent POF. The pathogenesis of POF is unclear, although genetic, physical, chemical, and immune factors can cause abnormal ovarian function (11-13).
In this study, we found that ovarian secretion of hormones was significantly reduced in patients with POF. In addition, the percentage of peripheral blood T lymphocytes in the $G_{1} / M$ period was significantly decreased $(\mathrm{P}<0.05)$. The above results are consistent with those of the study by Xie et al (14), which analyzed healthy women, women with POF, and women with ovarian reserve dysfunction. Their study demonstrated that the proportion of Treg cells in the blood of patients with POF was decreased $(\mathrm{P}<0.01)$. Furthermore, the levels of IFN- $\alpha$ in peripheral blood were significantly increased.

ROS refer to a series of oxygen free radicals including: $\mathrm{O}_{2}^{-}, \mathrm{H}_{2} \mathrm{O}_{2}, \mathrm{HO}_{2}$ and $\mathrm{OH}$. Middle and high concentrations of ROS caused cell apoptosis and necrosis induced by oxidative stress (15). With the progression of free radical biology, ROS have been shown to regulate the apoptosis and proliferation of certain tumor cells. In addition, our study found that patients in the experimental group (received melatonin treatment) had significantly decreased levels of plasma ROS and VEGF, whereas in the control group, there were no changes. Since it is structurally stable as a non-enzymatic antioxidant, MT is less prone to auto-oxidation. It can clear hydroxyl (-OH) groups, and different types of ROS, and plays a role in antioxidation.

Melatonin reduced the production of ROS. With both in vivo and in vitro experiments, previous studies reported that melatonin can inhibit hypoxia inducible factor-1 (HIF-1). HIF-1 is a transcription factor responsible for the expression of VEGF. Melatonin can reduce hypoxia induced angiogenesis. It was found that the ability of MT to clear -OH is more than 5 times than that of glutathione $(16,17)$. MT also has an indirect antioxidant effect, in that it can promote the expression of antioxidant defense enzymes by nuclear receptor mediated mechanisms, and enhance the activity of antioxidant enzymes (18-20).

Since anti-ROS mechanisms are involved in various pathophysiological processes, which generally require different inflammatory factors and multiple signaling pathways together 
to function, the results of the present study require further validation (19-21). The change in VEGF alone cannot explain the mechanism between POF and ROS. Further investigation is required to prove that decreasing ROS levels result in improved ovarian function (22).

Based on our results and those of other authors, we believe that reducing the levels of ROS with melatonin can regulate the T lymphocyte cycle and increase sex hormone levels in patients with POF. Melatonin may have clinical significance for the treatment of POF.

\section{References}

1. Blumenfeld Z and Evron A: Endocrine prevention of chemotherapy-induced ovarian failure. Curr Opin Obstet Gynecol 28 223-229, 2016.

2. Mishra B, Ortiz L and Luderer U: Charged iron particles, components of space radiation, destroy ovarian follicles. Hum Reprod 31: 1816-1826, 2016.

3. Roussev RG, Kaider BD, Price DE and Coulam CB: Laboratory evaluation of women experiencing reproductive failure. Am J Reprod Immunol 35: 415-420, 1996.

4. Wu X, Cai H, Kallianpur A, Li H, Yang G, Gao J, Xiang YB, Ji BT, Yu-Tang, Zheng $\mathrm{W}$ and Shu XO: Impact of premature ovarian failure on mortality and morbidity among Chinese women. PLoS One 2014;9:e89597

5. Tamura H, Nakamura Y, Korkmaz A, Manchester LC, Tan DX, Sugino $\mathrm{N}$ and Reiter RJ: Melatonin and the ovary: physiological and pathophysiological implications. Fertil Steril 92 328-343, 2009

6. Jang $\mathrm{H}$, Lee $\mathrm{OH}$, Lee $\mathrm{Y}$, Yoon $\mathrm{H}$, Chang EM, Park M, Lee JW, Hong K, Kim JO, Kim NK, et al: Melatonin prevents cisplatin-induced primordial follicle loss via suppression of PTEN/AKT/FOXO3a pathway activation in the mouse ovary. $\mathrm{J}$ Pineal Res 60: 336-347, 2016.

7. He H, Jiang DM, Kang B, Ma R, Bai L, Wang X and Zhao L: Gene expression profiling of melatonin receptor subtypes in the ovarian hierarchical follicles of the Sichuan white goose. Anim Reprod Sci 145: 62-68, 2014

8. Ma Q, Zhang T, Zhang P and Wang ZY: Melatonin attenuates postharvest physiological deterioration of cassava storage roots. J Pineal Res 60: 424-434, 2016.

9. Gurer-Orhan H, Karaaslan C, Ozcan S, Firuzi O, Tavakkoli M, Saso L and Suzen S: Novel indole-based melatonin analogues: evaluation of antioxidant activity and protective effect against amyloid $\beta$-induced damage. Bioorg Med Chem 24: $1658-1664,2016$.

10. Lohana CK and Samir N: Risk management of osteoporosis in postmenopausal women; a study of women in a teaching hospital. Glob J Health Sci 8: 55505, 2016.
11. Vriend $J$ and Reiter RJ: Melatonin and the von HippelLindau/HIF-1 oxygen sensing mechanism: a review. Biochim Biophys Acta 1865: 176-183, 2016.

12. Xu Y, Wang S, Jiang L, Wang H, Yang Y, Li M, Wang X, Zhao $\mathrm{X}$ and $\mathrm{Xie} \mathrm{K}$ : Identify melatonin as a novel therapeutic reagent in the treatment of 1-bromopropane(1-BP) intoxication. Medicine (Baltimore) 95: e2203, 2016.

13. Rhee YH and Ahn JC: Melatonin attenuated adipogenesis through reduction of the CCAAT/enhancer binding protein beta by regulating the glycogen synthase 3 beta in human mesenchymal stem cells. J Physiol Biochem 72: 145-155, 2016.

14. Xie JY, He W, Zhao LM, Chen M and Liang ZQ: The changes of CD4 ${ }^{-}$CD25 regulatory $T$ cells and changes of interferon gamma and expression of transforming growth factor beta 1 in patients with premature ovarian failure. West China Med J 3: 377-379, 2013 (In Chinese).

15. Bodas M, Van Westphal C, Carpenter-Thompson R, Mohanty KD and Vij N: Nicotine exposure induces bronchial epithelial cell apoptosis and senescence via ROS mediated autophagyimpairment. Free Radic Biol Med 97: 441-453, 2016.

16. Mehrzadi S, Kamrava SK, Dormanesh B, Motevalian M, Hosseinzadeh A, Hosseini Tabatabaei SM and Ghaznavi H: Melatonin synergistically enhances protective effect of atorvastatin against gentamicin-induced nephrotoxicity in rat kidney. Can J Physiol Pharmacol 94: 265-271, 2016.

17. Check JH, Wilson C, DiAntonio G and DiAntonio A: In vitro fertilization (IVF) outcome in women in overt menopause attempting to induce follicular maturation by follicle stimulating hormone (FSH) receptor down-regulation. Clin Exp Obstet Gynecol 43: 181-183, 2016.

18. Sundaresan NR, Marcus Leo MD, Subramani J, Anish D, Sudhagar M, Ahmed KA, Saxena M, Tyagi JS, Sastry KV and Saxena VK: Expression analysis of melatonin receptor subtypes in the ovary of domestic chicken. Vet Res Commun 33: 49-56, 2009.

19. Liu T, Li Q, Wang S, Chen C and Zheng J: Transplantation of ovarian granulosa-like cells derived from human induced pluripotent stem cells for the treatment of murine premature ovarian failure. Mol Med Rep 13: 5053-5058, 2016.

20. Kleszczyñski K, Zillikens D and Fischer TW: Melatonin enhances mitochondrial ATP synthesis, reduces ROS formation and mediates translocation of the nuclear erythroid 2-related factor 2 resulting in activation of phase-2 antioxidant enzymes (ã-GCS, HO-1, NQO1) in UVR-treated normal human epidermal keratinocytes (NHEK). J Pineal Res (In press) 2016.

21. Benedict C, Thom B, N Friedman D, Diotallevi D, M Pottenger E, Raghunathan JN and Kelvin JF: Young adult female cancer survivors' unmet information needs and reproductive concerns contribute to decisional conflict regarding posttreatment fertility preservation. Cancer 122: 2101-2109, 2016.

22. Laisk-Podar T, Lindgren CM, Peters M, Tapanainen JS, Lambalk CB, Salumets A and Mägi R: Ovarian physiology and GWAS: biobanks, biology, and beyond. Trends Endocrinol Metab 27: 516-528, 2016. 